\title{
Discrepancy of Karyotype and CMA/NGS in a PGD Patient With Cryptical Complex Chromosomal Rearrangement
}

Ting Wang

Guangdong Women and Children Hospital

Yinhuan Zhong

Guangdong Women and Children Hospital

Xianzheng Li

Guangdong Women and Children Hospital

Hanbiao Chen

Guangdong Women and Children Hospital

Jian Lu

Guangdong Women and Children Hospital

Weiwei Huang

Guangdong Women and Children Hospital

Chuangqi Chen

Guangdong Women and Children Hospital

Li Guo ( $\nabla$ guoli3861@163.com )

Guangdong Women and Children Hospital

\section{Research Article}

Keywords: Complex chromosome rearrangement, preimplantation genetic diagnosis, next generation sequencing, chromosome microarray analysis

Posted Date: June 24th, 2021

DOl: https://doi.org/10.21203/rs.3.rs-639547/v1

License: (c) (i) This work is licensed under a Creative Commons Attribution 4.0 International License. Read Full License 


\section{Abstract}

Introductions: Complex chromosome rearrangement (CCR) is a structural rearrangement involving more than two breakpoints. CCR carriers are at high risk for phenotypic abnormalities or reproductive failure, such as chromosomal abnormalities in fetuses and infertility. In this study, we presented a carriers with chromosome $(3,18)$ balanced translocation, whose fetus had duplications in chromosome 3 and deletions in chromosome 10 demonstrated by chromosomal microarray analysis (CMA).By revealing the cryptical translocation, we aimed to provide CCR carriers with more accurate risk assessment of abnormal pregnancy and better assisted reproduction with CMA and next generation sequencing(NGS).

Results: By using the high resolution of GTG-banding technology, a cryptical translocation in chromosome 10 was found and the karyotype of the carrier was revised as $46, \mathrm{XY}, \mathrm{t}(3 ; 10 ; 18)(\mathrm{p} 26.3 ; \mathrm{q} 26.1 ; \mathrm{q} 21.1)$.In the cycle of preimplantation genetic diagnosis (PGD),21 oocytes were retrieved, and 15 were fertilized. At last 7 embryos were biospied and sent to diagnosis by next generation sequencing(NGS). Unfortunately, none of the NGS results from the 7 biopsy embryos were normal. Combining previous literature and our results, we assessed the odds of a balanced embryo in a CCR carrier to be about $9.3 \%(28 / 302)$. The transferable embryo rate was approximately $71.4 \%(20 / 28)$ and healthy live born delivery rate was $55 \%(11 / 20)$.

Conclusions: NGS and CMA featured high automation, relatively low cost, high throughput, and high repeatability, which made them commonly used during prenatal diagnosis and PGD. The multiple technology combination can provide more accurate diagnosis and better fertility services for CCR patients.

\section{Introductions}

Complex chromosome rearrangement (CCR) is a structural rearrangement involving more than two breakpoints. The most common CCR involves three chromosomes and three breakpoints. Constitutional CCR are rare and approximately 250 cases had been reported so far[1]. The CCR occurrence in newborns was about 0.5\%[2].Balanced CCR carriers are prone to recurrent miscarriage, and unbalanced CCR is often associated with mental retardation and congenital malformations. In addition. CCR are not only associated with rare structural abnormalities, but occasionally occurs with gene deletion and duplication. These are mainly through the damage of dose-sensitive genes, cis-regulatory elements, so as to affect the expression of candidate disease genes through long-term positioning effect[3]. CCR carriers had a less than $6 \%$ chance of retrieving a normal/balanced blastocyst. The chance of getting pregnant is less than $4 \%[4]$. During meiosis, CCR can produce a large number of gametes and unbalanced chromosomes [5]. The application of preimplantation genetic diagnosis (PGD) to CCR carriers can reduce the risk of spontaneous abortion, thus producing fetuses with balanced chromosomes and avoiding the generation of embryos with unbalanced chromosomes[6].

The resolution of traditional karyotype analysis is limited, and it can only detect the abnormality of $>10 \mathrm{MB}$ fragments in general. Moreover, due to personal subjective judgment, it is easy to miss small structural changes. In recent years, with the development of molecular and cell biology technology, chromosome microarray analysis (CMA) and next generation sequencing(NGS) had been increasingly applied in prenatal diagnosis. CMA can cover the whole genome DNA, with high throughput, high resolution and fast detection speed[7].Schluth-Bolard $\mathrm{C}$ et al had proven that high-resolution NGS was effective in detailed studies of CCR[8].Constantia A et al found a cryptic breakpoint by whole-genome mate-pair sequencing in a CCR carrier, which was misdiagnosed by karyotype[9]. Frumkin T et al revealed a gain or loss at three chromosomes $(3,7,9)$ by PGS-CMA in six embryos, whose parents had a former diagnosis of normal karyotype[10].

In this study, we presented a carriers with chromosome $(3,18)$ balanced translocation, whose fetus had duplications in chromosome 3 and deletions in chromosome 10 demonstrated by CMA. By revealing the cryptical translocation, we aimed to provide CCR carriers with more accurate risk assessment of abnormal pregnancy and better assisted reproduction with CMA and NGS.

\section{Method}


A, 35-year-old, pregnant woman who had previously given birth to a normal child referred to our genetic medical center due to the high risk of noninvasive prenatal test(NIPT) performed in other hospital which demonstrated a deletion of 8Mb in chromosome(chr) 10. Chromosomal karyotype analysis at 400-band level suggested that the woman's husband had a significant balanced translocation on chr 3 and chr $8[46, X Y, t(3 ; 18)(p 26 ; q 21.1)]$, while a normal karyotype[46,XX] detected in the woman. In view of the inconsistency between the karyotype analysis and the NIPT results, we recommended a re-karyotype in our center. All methods were performed in accordance with the relevant guidelines and regulations. Informed consents were obtained from the couple before all examination and this case report was approved by the Ethics Committee of Guangdong Women and Children Hospital.

\section{Karyotype and CMA}

The couple accepted our suggestion and their peripheral blood was drawn in a vacuum heparin tube for phytohemagglutinin (PHA)-stimulated lymphocytes cultures. To obtain chromosomes with a resolution higher than 550 bands, we used thymine(Sigma, USA) and deoxycytidine(Sigma, USA) to synchronize the lymphocytes and inhibited chromosome contraction. According to the standard protocol, 20 metaphases were counted and five of them were karyotyped using lkaros system version 5.8.2(Zeiss, Germany). The International System for Human Cytogenomic Nomenclature (ISCN 2016) was employed to describe the karyotypes.

An amniocentesis was performed at 23 weeks gestation due to the high risk of NIPT.

$10 \mathrm{ml}$ of amniotic fluid were used for DNA extraction by QIAamp DNA Blood Mini Kit(QIAGEN, Germany), NANODROP 2000(Thermo USA) was applied to determine DNA concentration. CytoScan 750K chip (Affymatrix,USA) were used for CMA detection, including enzyme digestion, ligation, PCR amplification, purification, fragmentation, labeling, hybridization, washing, scanning.The CMA result was analyzed with ChAS3. 1.0.15 (Affymatrix USA) software. All the procedures were operated according to the manufacturer's protocol.

\section{NGS and PGD}

An IVF protocol was performed as described by Shen et al[11]. Controlled ovarian hyperstimulation was applied with gonadotropin-releasing hormone agonist long protocol. The cycle was followed by intramuscular injection of gonadotropin (Gn) after descending regulation, and the Gn dose was adjusted according to the follicle diameter monitored by ultrasonography. When the diameter of follicle was greater than $16 \mathrm{~mm}$, human chorionic gonadotropin (Serono, Switzerland) was injected for $6000 \sim 10000 \mathrm{u}$, and 21 oocytes were retrieved after $34 \sim 36 \mathrm{~h}$. The collectde oocytes were cultured in medium for 3 hours, those in metaphase II were fertilized by intracytoplasmic sperm injection (ICSI). The 15 fertilized oocytes were separately cultured in a complete medium (Chang, USA) at incubator (Thermo, USA) with $37^{\circ} \mathrm{C} 5 \% \mathrm{CO}_{2}$. After five days 7 high-quality embryos of 13 blastocysts were picked for blastocyst biopsy. Four to ten trophectodermal cells were biopsied from each of them using a $35 \mathrm{~mm}$ inner diameter biopsy micropipette (Humagen, USA). The biopsied cells were lysed and the whole genome amplification were performed with the SurPlex kit(Illumina, USA), including fragmentation, library preparation and amplification. All the 7 biopsied blastocysts were successfully amplified. A whole genome sequencing was performed by lon Torrent PGM (ThermoFisher, USA) platform according to the manufactor's protocol[12].

\section{Results}

The distal translocation of Chr10 was found to be involved in the husband's karyotype. Hence, the karyotype of the husband was revised as 46,XY,t(3;10;18) (p26.3;q26.1;q21.1).(Fig. 1), and the woman's re-karyotype was normal.The CMA result showed the fetus had 4.7Mb duplications in Chr 3 and 9.7Mb deletions in Chr 10 (Fig. 2). The couple decided to terminate the pregnancy because chromosomal structural abnormalities of the fetus involved many genes, which could lead to fetal malformation. PGD was advised to them by our genetic conselor on account of CCR.

Unfortunately, none of the NGS results from the 7 biopsy embryos were normal as Table.1 showed. NGS result of embryo no. 3 confirmed our previous CMA result and karyotype about Chr10's breakpoint(Fig. 3). The couple decided to abandon any embryo 
transfer and planned on next cycle.

\section{Discussions}

Reciprocal balanced translocations typically involved two chromosomal breakpoints, whereas CCR had three or more breakpoints. Theoretically, a balanced translocation carrier had only $1 / 9$ chance of delivering offspring with a normal phenotype. Due to the nature of CCR and the number of chromosomes involved, it was particularly difficult to analyze the meiotic behavior of CCR and its consequences. Therefore, there were relatively few data on meiotic separation of human CCR[13]. Previous studies had suggested that in the population with recurrent spontaneous abortion the frequency of CCR was $0.1 \%[14]$. The risk of offspring with an unbalanced karyotype was different in carriers of CCR. Natural pregnancy was discouraged in CCR carriers. Prenatal diagnosis was recommended even if a couple with CCR became pregnant naturally[15].

Preimplantation genetic diagnosis (PGD), provided to carriers of CCR, might reduce the risk of spontaneous abortion and the opportunity for offspring to carry chromosomal imbalances, and also could increased the chance of pregnancy by selecting against cleavage-stage embryos carrying unbalanced CCR[16]. The identification of CCR and the accurate description of breakpoints depended on the quality of chromosome analysis. Preliminary identification of CCR was performed using traditional cytogenetic methods based on GTG-banding and high-resolution karyotype. By introducing molecular cytogenetics technology (fluorescence in situ hybridization, FISH), the properties of CCR had been greatly improved. Various FISH methods for studying CCR had been tested, and a growing number of reports suggested that CCR may be more complex and common than initially thought $[9,10,16-19]$. However, FISH is limited to detecting specific chromosomes and requires a large number of probes to obtain reliable results. With the development of molecular technology, the application of PGD based on NGS provided an accurate method for detecting the unbalanced segmental rearrangement of embryos[4,10,20,21]. Unlike the traditional lowthroughput PCR technique, the next generation sequencing technology can comprehensively detect 23 pairs of chromosomes at high resolution. In addition, NGS can detect de novo copy number variants. High automation, high throughput, and high repeatability make NGS the most widely used technology in PGD. In this case we also employed CMA as control for comparison.

To our knowledge, this is the first study to summarize clinical outcome of PGD patients with CCR reported by previous literatures(Table 2). Combining previous literature and our results ( 0 balanced embryo out of a total 7 diagnosed in one PGD cycle), we assessed the odds of a balanced embryo in a CCR carrier to be about $9.3 \%(28 / 302)$, while the chance was $20-30 \%$ in the case of two chromosomal translocations[19]. The transferable embryo rate was approximately $71.4 \%(20 / 28)$ and healthy live born delivery rate was $55 \%(11 / 20)$ according to the data demonstrated in Table 2 . The couple was informed that the rate of balanced embryo acquired via PGD in CCR carrier is extremely low, and this should be taken into consideration in planning the next controlled ovarian hyperstimulation cycle.

In this case, the couple was re-karyotyped by reason of discrepancy in cytogenetic result and CMA. The cryptic translocation was finally found in chr10 with high resolution G-banding technique(550-bands level). This indicated that a high level of banding is necessary in cytogenetic examination. It could avoid misdiagnoses in initial consultation and reduce patient's dissatisfaction and unnecessary medical expenses. We did not conduct FISH verification on the breakpoint of chr10 for the reason as follow. First, customed FISH probes were expensive, which could add extra cost. Second, 550-bands level karyotype was sufficient to identify translocation on chr10 as demonstrated by Fig. 1. Third, we found the deletion of chr10 in the CMA results of the fetus, meanwhile there were chr10 gain or loss in 5 of the 7 biospied embryos according the NGS results(Table 1).

In conclusion, NGS featured high automation, relatively low cost, high throughput, and high repeatability, which made them commonly used during prenatal diagnosis and PGD. The combination of molecular and cytogenetic technology can provide more accurate diagnosis and better fertility services for CCR patients.

\section{Abbreviations}

CMA: chromosome microarray anlysis; NGS: next generation sequencing; PGD: preimplantation genetic diagnosis; CCR: complex chromosome rearrangement; Chr: chromosome; NIPT: noninvasive prenatal testing; GTG-banding: Giemsa trypsin banding; FISH: fluorescence in situ hybridization

Page 4/9 


\section{Declarations}

\section{Ethics approval and consent to participate}

This study was performed with the approval of Medical Ethics Committee of Guangdong Women and Children Hospital.

\section{Consent for publication}

Written consent for publication of medical data and genetic testing results was obtained from the affected couple.

\section{Availability of data and materials}

The datasets used and/or analyzed during the current study are available from the corresponding author on reasonable request.

\section{Competing interests}

The authors declare that they have no competing interests.

\section{Funding}

This study is supported by Guangzhou Basic and Applied Basic Research Project Grant 202102080233.

\section{Authors' contributions}

All authors have materially participated in the study and manuscript preparation.TW analyzed the clinical data, drafted the manuscript; LG and HC carried out the clinic data analysis, and participated in the design of the work; JL and WH participated in NGS and CMA analysis and conceiving the work. LG and HC participated in the karyotyping analysis. CC participated in the PGD.XL and YZ participated in the follow-up. All authors have approved the final article.

\section{Acknowledgements}

We would like to express our sincere gratitude to our patient and his family for their cooperation.

\section{References}

1. Madan K. Balanced complex chromosome rearrangements: reproductive aspects. A review. Am J Med Genet Part A. 2012;158A:947-63.

2. Durmaz CD, Yararbas K, Kutlay NY, Turedi O, Akin I, Gurbuz C, et al. Unusual chromosomal rearrangement resulted in interstitial monosomy 9p:case report. Cytogenet Genome Res 2016;148:19-24

3. Moralli D, Nudel R, Chan MTM, Green CM, Volpi EV, Benítez-Burraco A, et al. Language impairment in a case of a complex chromosomal rearrangement with a breakpoint downstream of FOXP2. Mol Cytogenet. 2015;8:36

4. Hu L, Wei Y, Luo K, et al. Clinical outcomes in carriers of complex chromosomal rearrangements: a retrospective analysis of comprehensive chromosome screening results in seven cases[J]. Fertility and Sterility, 2018:S0015028217320757.

5. Liedo B, Ortiz JA, Morales R, Manchon I, Galan F, Bernabeu A, et al. Characterization of a balanced complex chromosomal rearrangement carrier ascertained through a fetus with dup15q26.3 and del5p15.33: case report. Hum Fertil 2013;16:215-7.

6. Stern H. Preimplantation Genetic Diagnosis: Prenatal Testing for Embryos Finally Achieving Its Potential[J]. Journal of Clinical Medicine, 2014, 3(1):280-309.

7. Wallenborn $M$, Petters $O$, Rudolf $D$, et al. Comprehensive high-resolution genomic profiling and cytogenetics of human chondrocyte cultures by GTG-banding, locus-specific FISH, SKY and SNP array.[J]. European Cells \& Materials, 2018, 35:225241.

8. Schluth-Bolard C, Labalme A, Cordier M-PP, Till M, Nadeau G, Tevissen H,et al. Breakpoint mapping by next generation sequencing reveals causative gene disruption in patients carrying apparently balanced chromosome rearrangements with intellectual deficiency and/or congenital malformations. [J]J Med Genet. 2013;50:144-50.

Page 5/9 
9. Constantia $A$, Athina $T$, Andria $K$, et al.Cryptic breakpoint identified by whole-genome mate-pair sequencing in a rare paternally inherited complex chromosomal rearrangement[J]. Molecular Cytogenetics, 2018, 11(1):34.

10. Frumkin T , Peleg S , Gold V , et al. Complex chromosomal rearrangement-a lesson learned from PGS[J]. Journal of Assisted Reproduction and Genetics, 2017, 34(4):1095-1100.

11. Shen J, Cram D S, Wu W, et al. Successful PGD for late infantile neuronal ceroid lipofuscinosis achieved by combined chromosome and TPP1 gene analysis[J]. Reproductive Biomedicine Online, 2013, 27(2):176-183.

12. Hou Y , Fan W, Yan L , et al. Genome Analyses of Single Human Oocytes[J]. Cell, 2013, 155(7):1492-1506.

13. Pellestor F, Anahory T, Lefort G, Puechberty J, Liehr T, Hedon B, et al. Complex chromosomal rearrangements: origin and meiotic behavior. [J]Hum Reprod Update 2011;17:476-94.

14. Nonaka $T$, Ooki I, Enomoto $T$, et al. Complex chromosomal rearrangements in couples affected by recurrent spontaneous abortion[J]. Int J Gynecol Obstet, 2015, 128(1):36-39.

15. Liao Y, Wang L, Zhang D, Liu C.Identification of a balanced complex chromosomal rearrangement involving chromosomes 3 , 18 and 21 with recurrent abortion: case report. [J]Mol Cytogenet 2014;7:39

16. Vanneste $E$, Melotte $C$, Voet $T$, et al. PGD for a complex chromosomal rearrangement by array comparative genomic hybridization[J]. Human Reproduction, 2011, 26(4):941-949.

17. Zhang F, Carvalho CMB, Lupski JR. Complex human chromosomal and genomic rearrangements. Trends Genet 2009a;25:298-307

18. Escudero T, Estop A, Fischer J, Munne S. Preimplantation genetic diagnosis for complex chromosome rearrangements. Am J Med Genet A. 2008;146A(13):1662-9.

19. Lim CK, Cho JW, Kim JY, Kang IS, Shim SH, Jun JH. A healthy live birth after successful preimplantation genetic diagnosis for carriers of complex chromosome rearrangements. Fertil Steril.2008;90(5):1680-4.

20. Tan Y, Yin X, Zhang S, Jiang H, Tan K, Li J, et al. Clinical outcome of preimplantation genetic diagnosis and screening using next generation sequencing. [J]GigaScience 2014;3:30.

21. Brunet B C, Shen J, Cai L, et al. Preimplantation genetic testing for complex chromosomal rearrangement carriers by nextgeneration sequencing[J]. Reproductive Biomedicine Online, 2018, 37(3): 375-382.

\section{Tables}

Table 1 NGS results of seven embryos

\begin{tabular}{lll} 
Embryo ID & NGS results & outcome \\
\hline 1 & $47, \mathrm{XN},+18$ & no transfer \\
\hline 2 & $46, \mathrm{XN},-(3)(\mathrm{p} 26.3-\mathrm{p} 26.1)(4.8 \mathrm{Mb}),+(10)(\mathrm{q} 26.13-\mathrm{q} 26.3)(9.61 \mathrm{Mb})$ & no transfer \\
\hline 3 & $46, \mathrm{XN},+(3)(\mathrm{p} 26.3-\mathrm{p} 26.1)(4.8 \mathrm{Mb}),-(10)(\mathrm{q} 26.13-\mathrm{q} 26.3)(9.61 \mathrm{Mb})$ & no transfer \\
\hline 4 & $47, \mathrm{XN},+3,-(10)(\mathrm{p} 15.3-\mathrm{q} 26.13)(127.06 \mathrm{Mb}),-(18)(\mathrm{q} 21.1-\mathrm{q} 23)(31.90 \mathrm{Mb})$ & no transfer \\
\hline 5 & $47, \mathrm{XN},-(3)(\mathrm{p} 26.3-\mathrm{p} 26.1)(4.8 \mathrm{Mb}),+10,-(18)(\mathrm{p} 11.32-\mathrm{q} 21.1)(46.10 \mathrm{Mb})$ & no transfer \\
\hline 6 & $46, \mathrm{XN},-(10)(\mathrm{q} 26.2-\mathrm{q} 26.3)(6.76 \mathrm{Mb}),+(18)(\mathrm{q} 21.1-\mathrm{q} 23)(31.90 \mathrm{Mb})$ & no transfer \\
\hline 7 & $46, \mathrm{XN},+(18)(\mathrm{p} 11.32-\mathrm{q} 21.1)(46.10 \mathrm{Mb})$ & no transfer
\end{tabular}

NGS: next generation sequencing

Table 2 clinical outcome of PGD patients with CCR reported by our study and previous literature 


\begin{tabular}{|c|c|c|c|c|c|c|c|c|c|}
\hline literature & cycle & oocytes & $\begin{array}{l}\text { fertilized } \\
\text { oocytes }\end{array}$ & blastocyst & $\begin{array}{l}\text { biospied } \\
\text { embryos }\end{array}$ & $\begin{array}{l}\text { analyzed } \\
\text { embryos }\end{array}$ & $\begin{array}{l}\text { balance/normal } \\
\text { embryos }\end{array}$ & transfer & $\begin{array}{l}\text { healthy } \\
\text { live } \\
\text { born } \\
\text { delivery }\end{array}$ \\
\hline $\begin{array}{l}\text { Hu L et } \\
\text { al.(2018) }\end{array}$ & 8 & 145 & 110 & 51 & 47 & 46 & 3 & 2 & 2 \\
\hline $\begin{array}{l}\text { Brunet } \\
\text { BCFK,et } \\
\text { al(2018) }\end{array}$ & 7 & 84 & 66 & 25 & 25 & 25 & 6 & 4 & 4 \\
\hline $\begin{array}{l}\text { Frumkin } \\
\text { T et al. } \\
\text { (2017) }\end{array}$ & 2 & 42 & 33 & 14 & 14 & 14 & 2 & 2 & 1 \\
\hline $\begin{array}{l}\text { Vanneste } \\
\text { E et al. } \\
\text { (2011) }\end{array}$ & 2 & 26 & 18 & 16 & 16 & 16 & 4 & 2 & 0 \\
\hline $\begin{array}{l}\text { Lim CK } \\
\text { et al. } \\
\text { (2008) }\end{array}$ & 4 & 80 & 61 & 56 & 56 & 54 & 4 & 4 & 1 \\
\hline $\begin{array}{l}\text { Escudero } \\
\text { T et al. } \\
\text { (2008) }\end{array}$ & 13 & NM & NM & NM & 151 & 140 & 9 & 6 & 3 \\
\hline $\begin{array}{l}\text { our } \\
\text { study }\end{array}$ & 1 & 21 & 15 & 13 & 7 & 7 & 0 & 0 & 0 \\
\hline total & 37 & & & & 316 & 302 & $28 \varangle 9.3 \%{ }^{\mathrm{a}} \mathrm{\square}$ & $20\left(71.4 \%^{b}\right)$ & 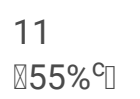 \\
\hline
\end{tabular}

PGD: preimplantation genetic diagnosis; CCR: complex chromosome rearrangement; NM: not mentioned; a=(balance/normal) embryos/analyzed embryos; b=transfer/(balance/normal) embryos; $c=$ healthy live born delivery/transfer

\section{Figures}



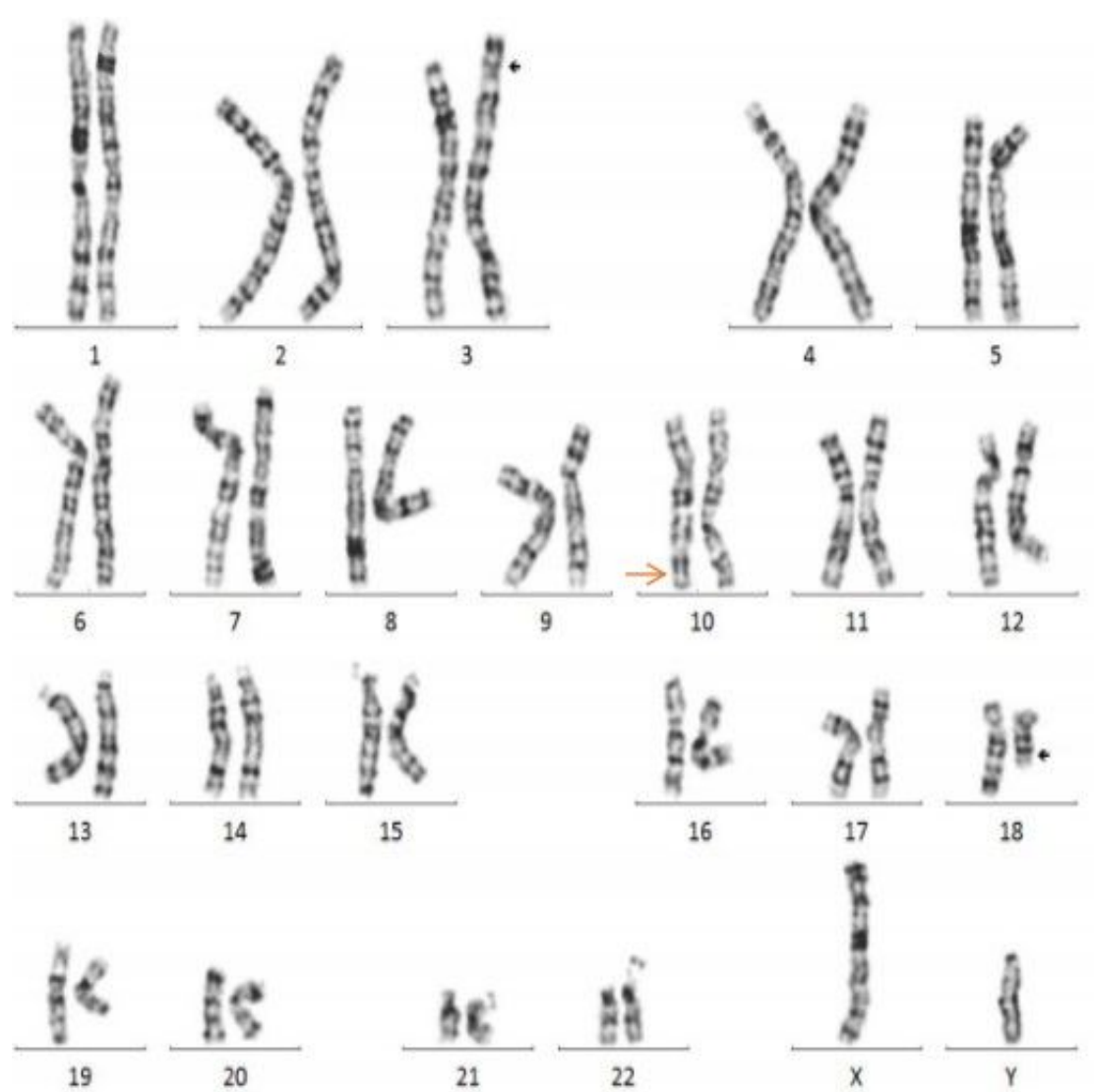

Figure 1

karyotpye of the husband in 550-bands level black arrow: apparent translocation; red arrow: cryptical translocation

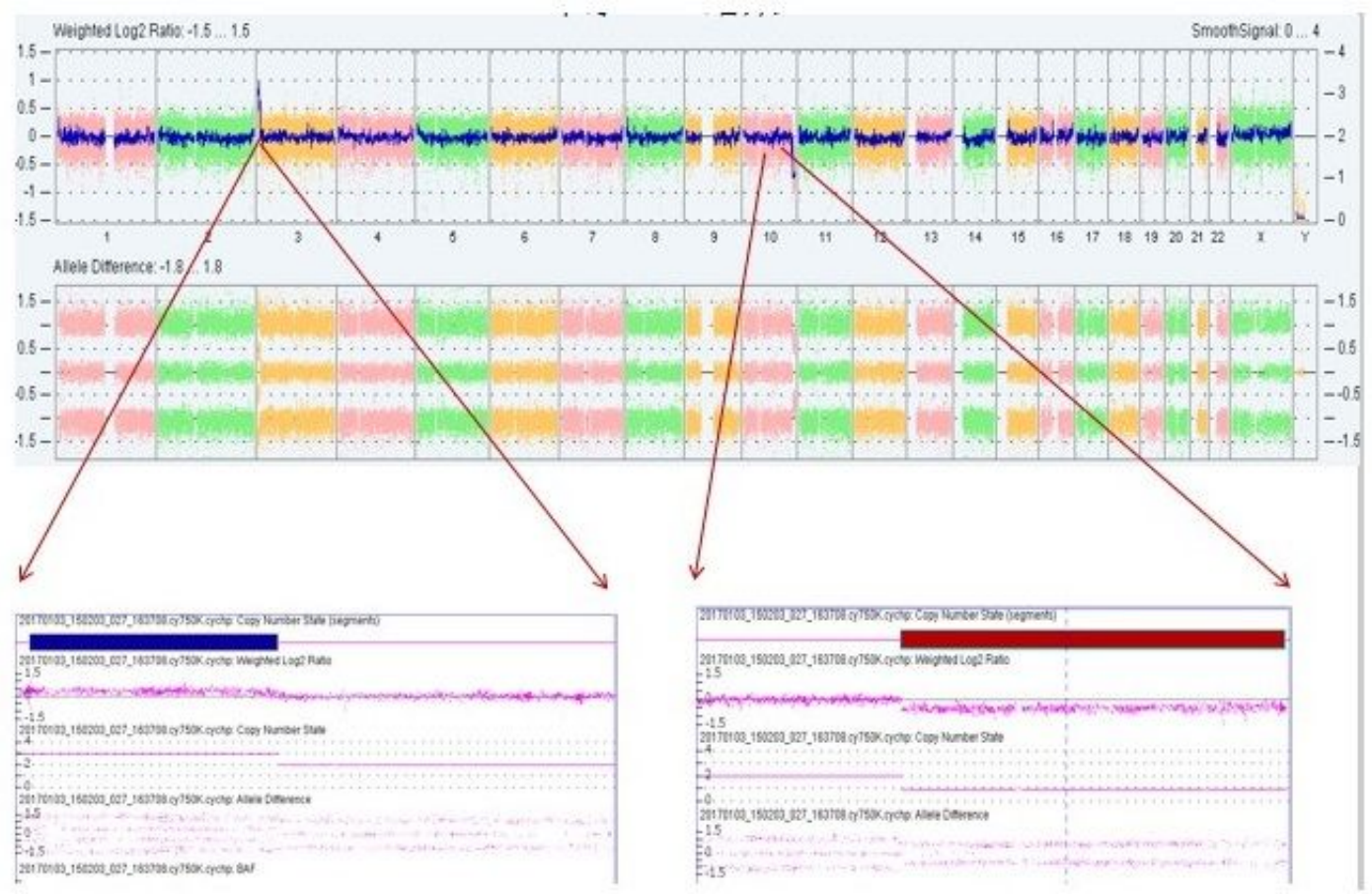

Figure 2

the CMA result of the fetus: arr[hg19]:3p26.3p26.1(61,891-4,742,752)×3,10q26.13q26.3(125,728,807-135,426,386)×1 


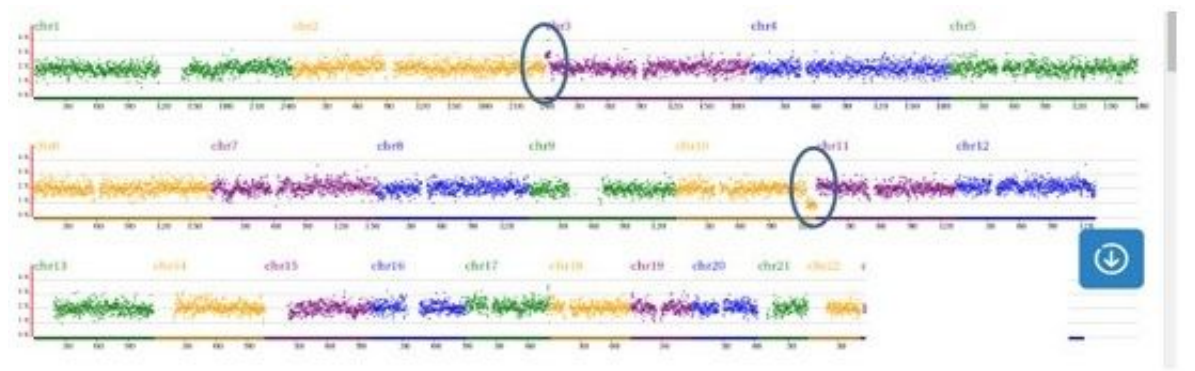

\section{Figure 3}

NGS result of embryo no. 3:+(3)(p26.3-p26.1)(4.8Mb),-(10)(q26.13-q26.3)(9.61Mb) 